\title{
Morphology of air invasion in an immersed granular layer
}

\author{
Germán Varas, Valérie Vidal, and Jean-Christophe Géminard \\ Laboratoire de Physique, Université de Lyon, Ecole Normale Supérieure - CNRS, 46 Allée d'Italie, F-69364 Lyon Cedex, France
}

(Received 19 April 2011; published 23 June 2011)

\begin{abstract}
We report a study of the paths formed by a finite volume of air gently injected at the base of an immersed granular material. A two-dimensional model, based on experimental observations, shows that the typical height and width of the region explored by the branched path depends not only on the injected volume $V$, but also on a dimensionless parameter $\chi$ which accounts for the relative effects of the gravity and capillarity. For a given injected volume $V$, larger gravity effects lead to taller and narrower structures; for a given $\chi$, the typical height and width of the structure scale like $V^{1 / 2}$ and $V^{1 / 4}$, respectively, while the typical gaseous fraction in the corresponding region increases accordingly like $V^{1 / 4}$. Such results can be of practical importance: For instance, gas can be trapped on purpose in an underground natural container below a granular slurry. Our results can help in predicting if the gas is likely to reach the free surface and escape the system if the container presents a defect (hole or fracture).
\end{abstract}

DOI: 10.1103/PhysRevE.83.061302

PACS number(s): 83.80.Fg, 47.57.Gc, 47.85.Dh

\section{INTRODUCTION}

The invasion of a gas in porous media is encountered in a wide range of systems, from industrial processes (oil industry $[1,2]$, methane hydrate dissociation [3], etc.) to geophysical phenomena [4-7]. A typical example of the application is air sparging [8]: air is injected into the subsurface below the lowest known depth of contamination and, due to buoyancy, air serves to remove or helps degrade the contaminants. Concerning natural processes, the study of gas emission at the sea floor (venting dynamics) has led to the study of the release of methane from pockmarks in the mid-Atlantic continental shelf [9]. These studies contributed to the understanding of the carbon-cycle perturbations. The results are important because these pertubations are likely to induce global climate changes [10].

Because of their implication in a wide range of systems and because of the potentially huge economical benefits, the understanding of such invasion processes has attracted the scientific community. They resemble many growth processes such as the Eden cluster model [11], ballistic models [12,13], dendritic growth, or the diffusion-limited aggregation (DLA) [14]. These models, which have been intensively studied $[15,16]$, especially in numerical simulations, consider a homogeneous media in the absence of external forces [17].

One can also mention various numerical studies of similar systems, from the destabilization produced by gravity in two-dimensional (2D) porous media [18-20] to the study of the fractal dimension in an etched network [21]. Most of the systems involve tip effects in which the local growth velocity is proportional to the local gradient of an external field (pressure, impurity concentration, etc.). We also point out that a very similar growth or propagation phenomenon is observed in the mining industry when fragments are extracted from an extraction point at the base of the ore bed (draw body [22]).

Concerning, in particular, the injection of a gas in granular materials, we can distinguish the biphasic case (grains and air) [23] from the triphasic case (grains, fluid, and air) $[24,25]$, the latter being more complex and not fully understood. The dynamics of the interface between air and an immersed granular material has been experimentally studied in a Hele-Shaw cell [26], mainly in a regime in which the gas forms a finger, reminiscent of the Saffman-Taylor finger. In this regime the grains are displaced by the interface. The main physical mechanisms at play involve the surface tension, the viscous dissipation, and a pressure gradient. The morphology produced during the air injection can be influenced by the rate of gas delivery and the vertical distance from the source [27]. We lately showed that, in a regime in which the air creates a path between the grains without moving them, the region invaded by the gas can be described as the result of a diffusion process [24]. The first simplistic approach neglected the formation of side branches during the growth of the air path. Here we propose a numerical analysis, based on experimental observations, of the role played by the injected volume and by the properties of the granular bed in the morphology of the region invaded by the gas, taking into account the formation of side branches.

\section{PROBLEM STATEMENT}

We aim at characterizing the geometry of the region invaded by a gas locally injected at the base of an immersed granular bed. In Ref. [24], we reported experimental results obtained in both three-dimensional (3D) and 2D experimental setups. The 2D system proved to be very useful as it makes it possible to visualize the paths created by the gas within the granular matrix. Thus, we will limit the present study to experimental examples obtained in a thin Hele-Shaw cell and numerical results from a (2D) model. We were previously interested in the loci of the gas emission at the free surface resulting from a continuous injection of gas. Here, we consider a qualitatively different situation in which a finite amount of gas is injected so that the invaded region does not reach the free surface. We aim at characterizing its morphology.

\section{A. Summary and limitations of the former results}

Long-term experiments [24] showed that, for the (2D) cell, the invasion of the immersed granular bed by the gas can be interpreted in terms of a diffusive-like model, the system exhibiting at long times a parabolic fluidized region 
whose width $w$ depends on the vertical distance $z$ from the lower edge as $w=\sqrt{D z}$. We proposed that the diffusion coefficient $D$ was an increasing function of a unique parameter of the system $\chi \equiv \sigma_{P} / \rho g d$, which compares the width $\sigma_{P}$ of the distribution of the capillary overpressure associated with the passage between the grains with the typical hydrostatic pressure variation $\rho g d$ over the grain size $d$. The result can be understood as follows: For very small grains, the hydrostatic pressure difference is very small compared to the capillary overpressure which scales like $1 / d$ (thus much smaller than the width of its distribution) and, locally, the air creating its path between the grains propagates as if inside an isotropic medium. The width of the invaded region is large, which corresponds to large $D$. On the contrary, if the effect of the gravity is much larger than the capillary overpressure, the growth of an air finger in the system is not limited by the capillarity and the gas crosses the system straight along the vertical (very large grains). The invaded region is narrow, which corresponds to small $D$.

The previous experimental results are worth extending in several aspects. First, we were not able to check experimentally the dependence of $D$ on $\chi$. Indeed, one can barely vary $\chi$ in a well-controlled manner by changing the grain size $d$ because the polydispersity of the samples and the packing of the grains are difficult to control. Second, in the theoretical approach, we assumed that the air was creating paths without side branches, which is obviously not the case when the effects of the gravity are weak. Finally, from a practical point of view, the geometry of the region invaded by a constant volume of gas is, at least, as interesting as the loci of the gas emission at the free surface in the continuous regime. Thus, we aim here at extending the previous results to branched invasion paths in the case of the injection of a finite volume.

\section{B. Preliminary experimental results}

To directly observe the paths of air within the granular material, we designed a 2D setup that consists of a Hele-Shaw cell (Fig. 1): the granular matter, immersed in water, is contained between two vertical walls (glass plates $40-\mathrm{cm}$ wide, $30-\mathrm{cm}$ high, gap $2 \mathrm{~mm}$ ). To control the gravity effects, the cell can be tilted by an angle $\alpha$ with respect to the vertical so as to produce an effective gravity $g_{\text {eff }} \equiv g \cos \alpha$. The experimental setup thus makes it possible to change $\chi$, the granular material remaining unchanged. The injection of air through an inlet located at the center of the lower edge is insured by a mass-flow controller (Bronkhorst, Mass-Stream Series D-5111). The flow rate $\Phi$ can be tuned in the range 0.2 to $2.2 \mathrm{~mL} / \mathrm{s}$. The granular material consists of glass beads (USF Matrasur, sodosilicated glass) previously sieved to control their size (diameter $d=150-250,250-425$, and 425-600 $\mu \mathrm{m}$ ). A webcam (Logitech, QuickCam S7500, $640 \times 480 \mathrm{px}^{2}$, $20 \mathrm{images} / \mathrm{s}$ ) is used to image the system from the side, whereas the light source consists of a transparency flat viewer (Just NormLicht, Classic Line) positioned behind the cell. The initial state of the system consists of an immersed granular bed (typical depth $24 \mathrm{~cm}$ ), free of bubbles, whose surface is leveled. A chosen volume of air $V$ is then gently injected in the system.

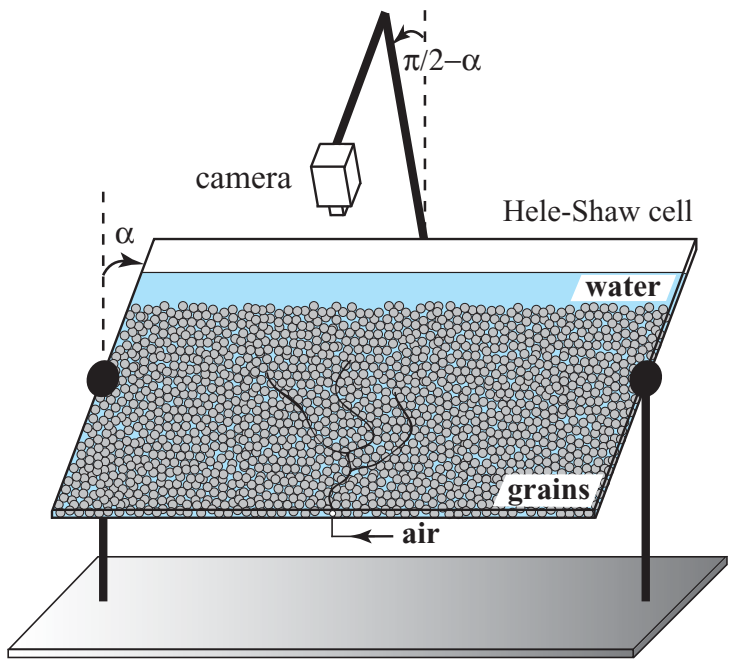

FIG. 1. (Color online) Sketch of the experimental setup. A total volume $V$ of air is injected at constant flow rate $\Phi$ into a thin vertical cell (Hele-Shaw cell) containing a granular layer immersed in water. The effective gravity is changed by tilting the cell by an angle $\alpha$ (from $0^{\circ}$ to $90^{\circ}$ ). The formation of the pattern in the granular bed is recorded with a camera positioned in front of the experimental cell.

The preliminary experimental results (Fig. 2) show that the branches are more numerous and, thus, the pattern more compact when the effective gravity is reduced by tilting the experimental cell. Accordingly, the invaded region is broader and smaller in this case whereas an increase of $g_{\text {eff }}$ leads to a narrower and taller pattern with less branches. To characterize the average geometry of the invaded region we would have to repeat the experiment a large number of times, which is difficult. Indeed, once the pattern is formed, to reset the initial condition, one must open the cell to remove the air trapped in the system, which takes a long time. Moreover, we would not be sure to prepare the system in the exact same way and the state of the granular packing (its density, for instance) might be different from one run to another.

Thus, to overcome the difficulty, we performed the numerical analysis of the problem that is thoroughly described in Sec. III.

\section{NUMERICAL ANALYSIS}

To assess the dependence of the geometry of the invaded region on the problem parameters, especially $\chi$ and the injected volume $V$, we perform the simple numerical analysis whose ingredients and results are reported in the Secs. III A and III B, respectively.

\section{A. Ingredients}

In a simplified 2D approach of the system, we consider that the granular packing reduces to a $2 \mathrm{D}$ square network, each vertex corresponding to the void space between four grains. The size of the network $(1200 \times 1000)$ compares with the typical size of the experimental cell in units of grains (typically 1600 grains in width by 1200 grains in height) and is large enough to avoid boundary effects on the sides and at the top. 

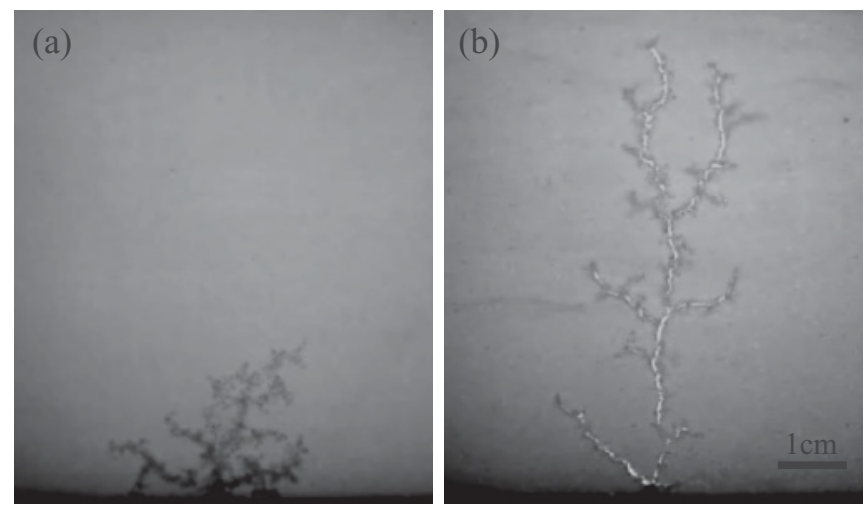

FIG. 2. Images of the experimental pattern. The 2D experiment reveals that, for the same volume $V=1.98 \mathrm{~mL}$, the path created by the injected gas has a drastically different geometry depending on the effective gravity $g_{\text {eff }}$. (a) For an almost horizontal cell $\left(\alpha=80^{\circ}\right)$, one observes a highly branched pattern, which remains located around the outlet (note that its typical width compares with its typical height). (b) By contrast, for a vertical cell $\left(\alpha=0^{\circ}\right)$, even if the image reveals a significant number of branches, the maximum height reached by the gaseous structure is much larger than its width $(d=250-425 \mu \mathrm{m})$.

The only boundary condition is that the gas cannot trespass on the bottom plane.

The propagation of an air finger in the material is limited by the capillary overpressure $\delta P_{c}$ to overcome going from one vertex to a neighboring one. Considering that the typical size of the pass between the two void spaces scales like the grain diameter $d$ [25], we estimate that, on average, $\delta P_{c} \sim \gamma / d$, where $\gamma$ denotes the air-water surface tension. However, due to the local heterogeneity of the system (polydispersity, wetting conditions, local arrangement of the grains, etc.), $\delta P_{c}$ differs from one pass to another. Thus, to account for the heterogeneity, we assume that the links between the vertices are associated with capillary overpressures distributed according to a Gaussian distribution of width $\sigma_{P}$ around the nominal value $\Delta P_{c}$ which, we remind, is of the order of $\gamma / d$.

The propagation of the air finger along the vertical is favored by the additional contribution of the hydrostatics. Indeed, in the experiments, the water that fills the space between the grains is subjected to gravity so that, for instance, the pressure difference in the water between one vertex and the first neighbor above is $\delta P_{g}=\rho g_{\text {eff }} d$, where $\rho$ denotes the density of water. In the numerical computations, the effects of the gravity are accounted for by considering that the threshold overpressure to overcome to go from one vertex to another is $\delta P_{t}=\delta P_{c}-\rho g_{\text {eff }} z$, where $z$ (positive) denotes the vertical distance from the outlet plane (bottom edge). Technically, we associate with each of the links the threshold overpressure $\delta P_{t}$ by adding the corresponding contribution of the gravity to the previously chosen map $\delta P_{c}$.

The air path is calculated as follows: First, the finger is grown from the virtual outlet, at the center of the bottom edge. Second, during the finger growth, from an already existing path, we consider the whole set of links connected to vertices occupied by the gas and determine the one corresponding to the smallest value of the threshold overpressure $\delta P_{t}$. We make the air invade the corresponding vertex, by adding the latter to the air path. The procedure thus allows the generation of

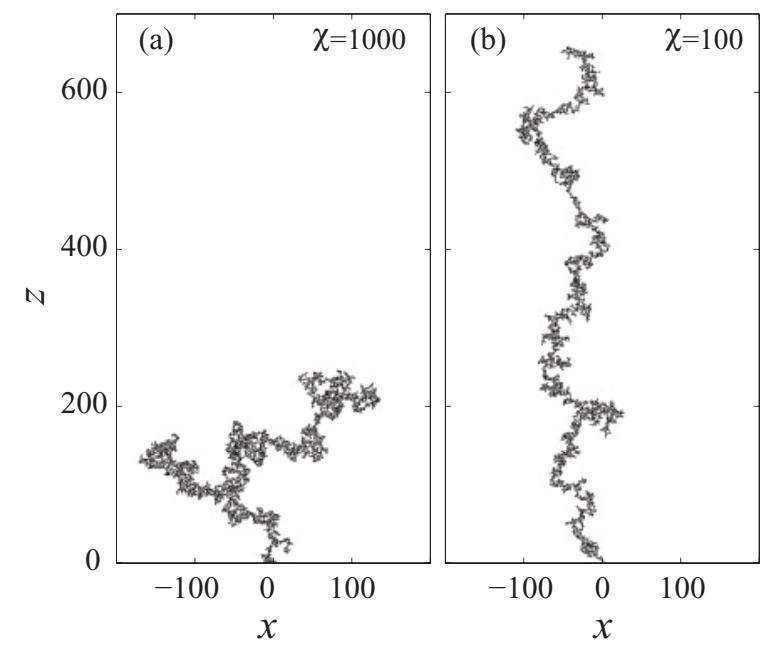

FIG. 3. Air paths from the numerical simulations. Note first that the numerical procedure allows the creation of side branches. For the same injected volume, thus the same number $N=4000$ of occupied vertices, one observes that the geometry of the path depends drastically on the control parameter $\chi[$ (a) $\chi=1000$; (b) $\chi=100]$. As expected and also observed experimentally, for smaller $\chi$ (larger gravity effects) the finger exhibits less branches and reaches a larger height.

branches (Fig. 3). We repeat the process until a chosen number of vertices $(N=2000,4000$, and 8000$)$, corresponding to a chosen volume $V \equiv N v_{p}\left(v_{p}\right.$ is the typical volume associated with the void space between the grains), are occupied. Finally, to get a relevant estimate of the average geometry of the invaded region, we repeat the whole process 2000 times for the same parameters (for a given $\chi$ and $N$ ).

\section{B. Results}

In the present section, we discuss the geometrical properties of the region invaded by the gas. The discussion is based on averages of 2000 numerical paths (Fig. 4).

\section{Geometrical characteristics of the invaded region}

As expected, for $\chi=\infty$ [Fig. 4(a)], the gas invades the granular packing in an almost isotropic manner, the paths filling half a disk above the outlet. The only anisotropy results from the boundary condition (no air flow) at the lower edge of the system. The observed pattern resulting from a pure diffusive process with a reflecting boundary the typical size of the invaded region scales like $d \sqrt{N}$. When $\chi$ is decreased, and thus the pressure gradient increased, the invaded region elongates along the vertical. However, one can notice that, for a given injected volume, the width of the structure does not change significantly whereas its height significantly increases [Figs. 4(b)-4(e)].

To report a qualitative behavior of the system, we first determine the contours of the invaded region associated with the isodensity lines in Fig. 4: A contour is defined by the fraction $f$ such that, over 2000 paths, the contour is locally crossed $2000 f$ times [Fig. 5(a)]. 


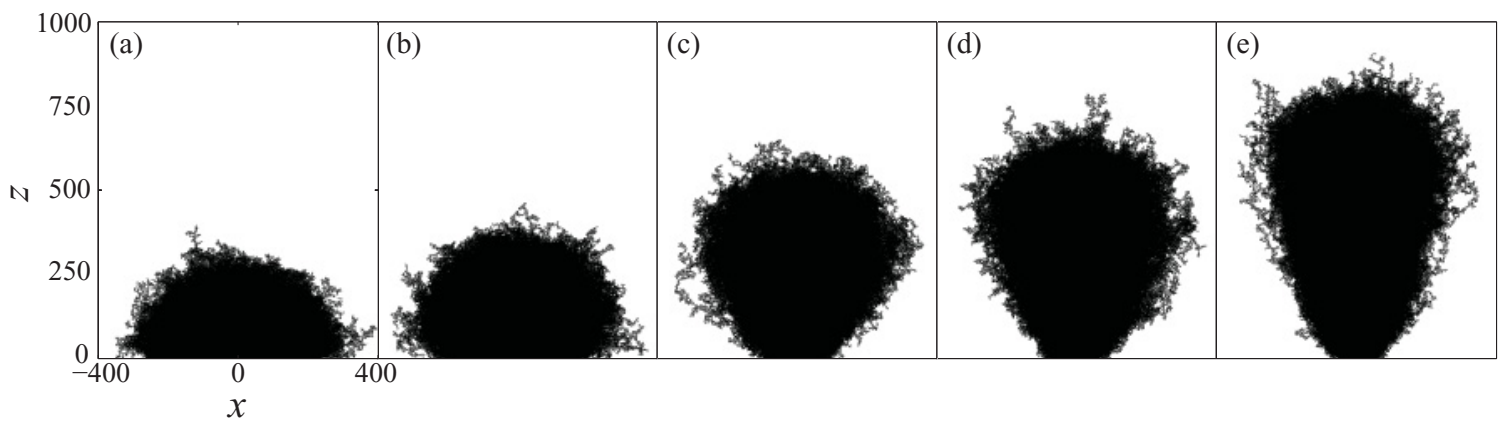

FIG. 4. Superposition of 2000 paths for the same injected volume $V$ and different values of $\chi$. The morphology of the invasion region goes from an almost circular pattern for a horizontal cell [(a) zero effective gravity, $\chi=\infty$ ] to a vertically elongated shape when $\chi$ is decreased $[N=8000$, (a) $\chi=\infty$; (b) $\chi=1250$; (c) $\chi=250$; (d) $\chi=166$; (e) $\chi=100]$.

A quantitative characterization of the structure geometry is provided by measurements of its width $\Delta x$ and height $\Delta z$. From the superposition of 2000 paths [Fig. 5(a)], we consider the intensity profile along the $x$ axis, estimated over the whole height of the system [Fig. 5(b)]. As expected, the profile is symmetric and we consider $\Delta x$ to be the width at half the peak value. In the same way, we consider the intensity profile along the $z$ axis, estimated over the whole width of the system [Fig. 5(c)]. We observe that the profile is almost flat and
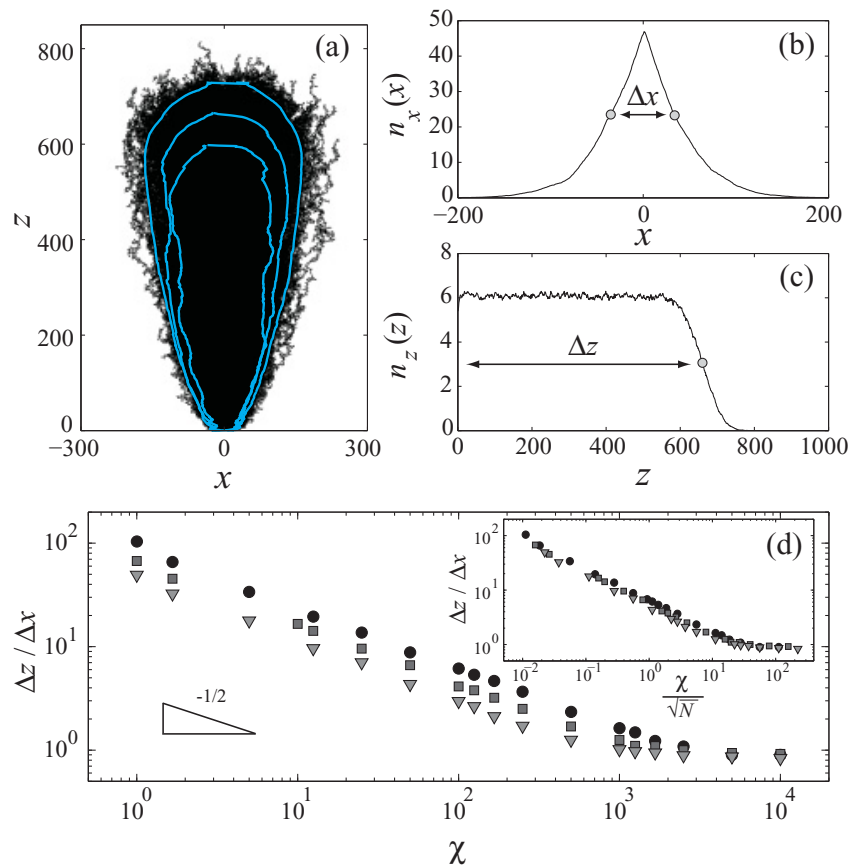

FIG. 5. (Color online) Contours, width $\Delta x$, and height $\Delta z$ of the invaded region and aspect ratio $\Delta z / \Delta x$ vs. $\chi$. (a) We report the contours (lines) for $f=2,4$, and $6 \%$ (over 2000 paths, $\chi=25$ and $N=4000$ ). (b) The width $\Delta x$ is defined to be the width at half height, over the entire pattern, of the intensity profile along the $x$ axis. (c) The height $\Delta z$ is that of the point at half the plateau value, over the entire pattern, of the intensity profile along the $z$ axis. (d) Aspect ratio $\varrho \equiv \Delta z / \Delta x$ vs. $\chi$ for three values of the injected volumes $(\boldsymbol{\nabla}: N=2000, \boldsymbol{\square}: N=4000$ and $\bullet: N=8000)$. Inset: A collapse of the measurements is observed when reporting the aspect ratio as a function of $\chi / \sqrt{N}$. suddenly decreases above a given altitude. We define $\Delta z$ as the altitude of the point corresponding to half the plateau value.

\section{Dependence on $V$ and $\chi$}

Reporting the contours of the invaded region for various values of the injected volume (various $N$ ) and effective gravity (various $\chi$ ), one observes that, qualitatively, the geometry of the pattern highly depends on both control parameters. First, one observes that, for increasing injected volumes [Fig. 6(a)], the height $\Delta z$ increases almost linearly with $N$. We observe that $\Delta z$ increases faster than the average width $\Delta x$, which results in an increase of the aspect ratio $\varrho$ [Fig. 5(d)]. Second, for a given injected volume [Fig. 6(b)], a decrease in $\chi$ results in an increase in the height $\Delta z$ and in a decrease in the width $\Delta x$, which result in a drastic increase in $\varrho$. Note that, when $\chi$ is decreased the shape of the invaded region goes from a half-disk to a parabola or cone. We expect that the air goes straight up through the system for $\chi \ll 1$.

The quantitative changes in the shape of the invaded region can be assessed by reporting $\varrho$ as a function of $\chi$ [Fig. 5(d)]. For the whole set of experimental data, we observe that
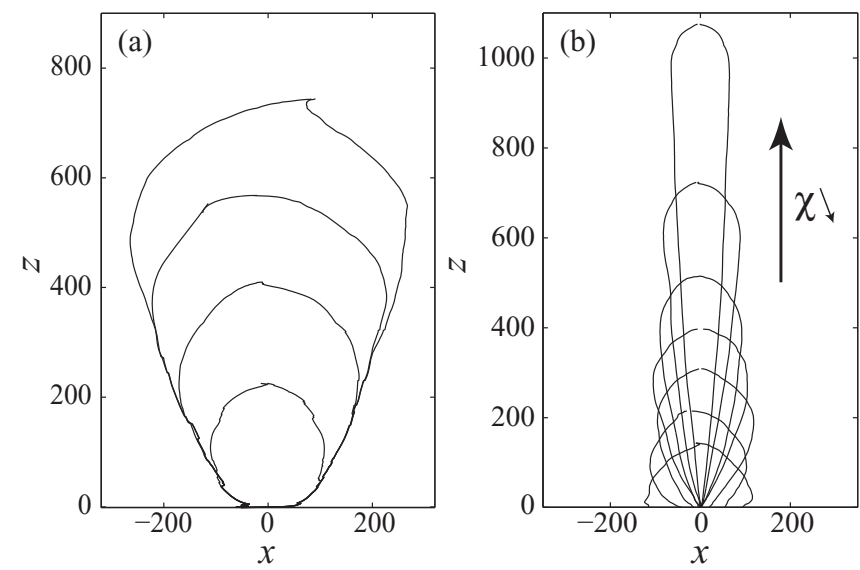

FIG. 6. Shape of the invaded region vs. $N$ and $\chi$. Contours are reported on average over 2000 paths for $f=2 \%$. (a) Contours for increasing volumes $(N=2000,4000,6000$, and 8000) for $\chi=125$. (b) Contours for decreasing effective gravity $(\chi=1.6,5.0,12.5,25,50,100, \infty)$ for the same injected volume $(N=2000)$. 

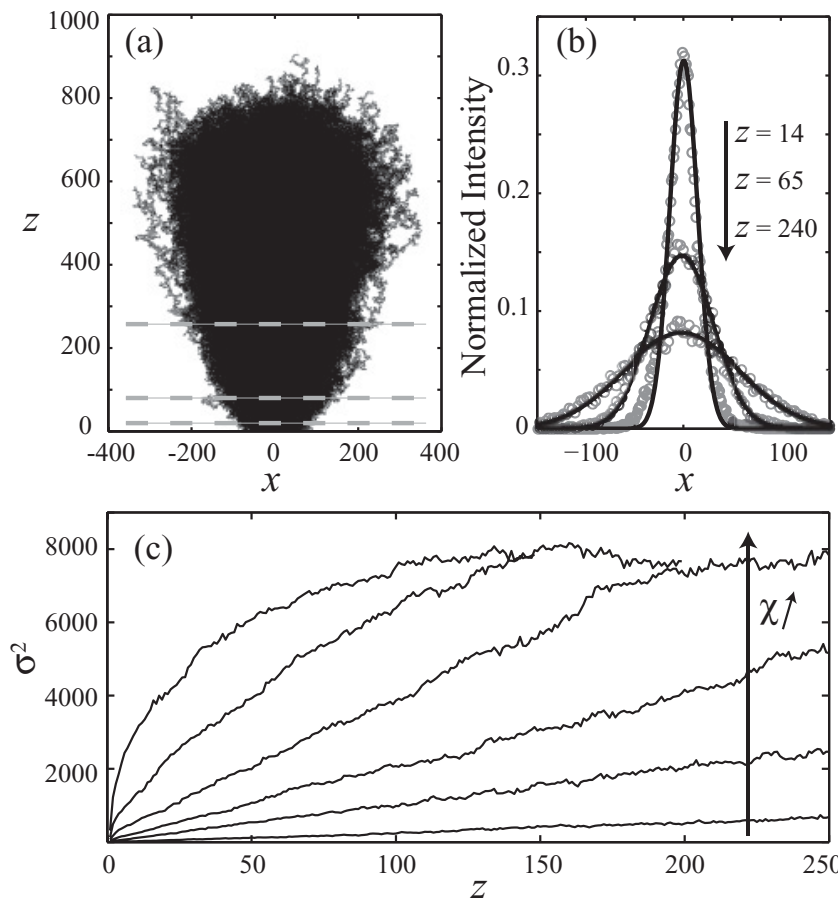

FIG. 7. Analysis of the probability profiles. (a) Superposition of 2000 paths for $\chi=166$. The gray horizontal lines are associated to the profiles reported in the right panel. The dashed gray lines represent three different heights. (b) Profiles of the probability density at three different altitudes $z=14,65$, and 240. (c) Square $\sigma^{2}$ of the distribution width as a function of the altitude $z$ for $\chi=5,50,166,500,1666$, and $\infty$. The system exhibits a diffusive behavior for small $\chi$. The curvature of the invaded region near the outlet is obtained from the slope at $z=0(N=8000)$.

$\varrho \propto 1 / \sqrt{\chi}$ in a wide range of $\chi$ and reaches a plateau value of the order of the unity $\varrho \simeq 0.82$ for large $\chi$. For a compact structure (isodensity), one would expect the aspect ratio to tend to 1 in the absence of gravity, in the absence of a lower boundary. Taking into account that the density is not constant within the structure and that the air flow is limited by the lower edge, we are not thus surprised that the ratio tends to a value of the order of the unity, but not exactly to 1 . Interestingly, we note a collapse of the measurements when reporting the $\varrho$ as a function of $\chi / \sqrt{N}$ : the scaling $\varrho \propto$ $1 / \sqrt{\chi}$ holds true for $\chi \lesssim 30 \sqrt{N}$ whereas $\varrho \simeq 0.82$ for larger values.

Additional pieces of information about the invasion process can be obtained by considering in Fig. 7(a) the probability density along $x$ for a given altitude $z$ : From the superposition of 2000 paths, one obtains the number of passages at a distance $x$ from the axis, for a given altitude $z$, by considering the corresponding density profiles [Fig. 7(b)]. From the profiles, one gets the typical width $\sigma$ of the region crossed by the air paths as a function of $z$ [Fig. 7(c)]. We observe that, for small enough values of $\chi$ and $z, \sigma^{2}$ is proportional to $z$, which accounts for the parabolic shape of the invaded region near the outlet. By extension, whatever the value of $\chi$, the shape of the invasion pattern near the outlet can be accounted for by its radius of curvature, equivalent to an effective diffusion coefficient $D_{\text {eff }} \equiv \partial \sigma^{2} /\left.\partial z\right|_{z=0}$ [Fig. 8(a)]. We obtain
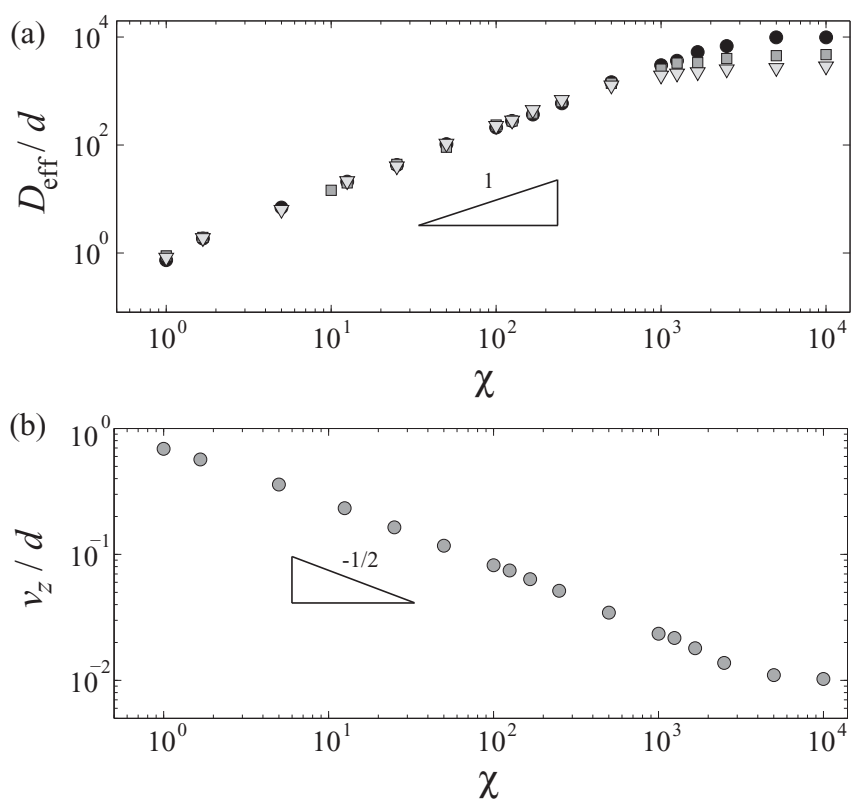

FIG. 8. Coefficient $D_{\text {eff }}$ and velocity $v_{z}$ vs. parameter $\chi$. The radius of curvature of the invaded region, near the outlet, is independent of the injected volume $(\boldsymbol{\nabla}: N=2000, \boldsymbol{\square}: N=4000$, and $\bullet: N=8000)$ and is given by $D_{\text {eff }} \simeq d \chi$ provided that the gravity effects are large enough $(\chi \lesssim 30 \sqrt{N})$. In the same conditions, the typical vertical size of the structure $\Delta z$ increases linearly with $N$. The velocity (the slope) $v_{z}$ scales like $d / \sqrt{\chi}$, the prefactor being of the order of unity.

that $D_{\text {eff }} \simeq d \chi$ for, again, $\chi \lesssim 30 \sqrt{N}$. Finally, to complete the description of the invasion pattern, one must consider the relation between the height $\Delta z$ and $N$ (the injected volume). For large enough injected volumes (large $N$, i.e., $\chi \lesssim 30 \sqrt{N}$ ), $\Delta z$ depends linearly on $N$ so that one can define the typical vertical growth velocity $v_{z} \equiv d \Delta z / d N$. One observes that, numerically, $v_{z} \propto d / \sqrt{\chi}$, the prefactor (about 0.8) being of the order of unity [Fig. 8(b)].

\section{DISCUSSION AND CONCLUSION}

Contrary to classical growth models [11-14], our model takes into account both a heterogeneous medium (capillary overpressure distribution) and the effect of an external field (gravity). Previous studies of fluid invasion in a 2D porous medium, including the gravity destabilizing effect, focused on the geometry of a single invasion pattern $[18,19]$. By contrast, our work aims at characterizing the morphology of the region potentially explored by the invading fluid. The numerical results show that, as expected, the shape of the invaded region depends drastically on $\chi$ (i.e., on the effective gravity). Less obvious, an increase of $N$ (i.e., of the injected volume) does not simply lead to a dilation of the invaded region, but to a change in $\varrho$ (i.e., in the geometry).

The present numerical model exhibits a much richer behavior than that exhibited by our previous analytical analysis [24]. Indeed, formerly, the side branching and the 
return of the air path to a previous lower position were not allowed. The path was forced to grow upward. The main difference in the results is that, formerly, the radius of curvature (or effective diffusion coefficient) $D_{\text {eff }}$ was predicted to be a complex function of $\chi$ whereas the prediction of the present numerical results is that $D_{\text {eff }} \simeq d \chi$ for $\chi \lesssim 30 \sqrt{N}$. In addition, we report that the vertical size of the structure is linear as a function of the injected volume $V$ and we estimate the dependence of the associated velocity on the control parameter $\chi, v_{z} \simeq 0.8 d / \sqrt{\chi}$.

It is particularly interesting to interpret the parameter $\chi \equiv \sigma_{P} / \rho g d$, which compares the width of the distribution of the capillary overpressure within the pores to the variation of the hydrostatic pressure over the grain size. On the one hand, for a given injected volume $N$, the air inside the existing path being connected, one can consider the path as an isobar. On the other hand, the pressure in the liquid, outside the air path, increases from the path tip (its highest point) downward, which makes the formation of the side branches more and more difficult at depth, below the path tip. Considering the meaning of $\chi$, one can estimate that side branches cannot form at a distance larger than $d \chi$ below the tip. As a consequence, the gas injection results either in the formation of side branches in a region of typical height $d \chi$ (in a local increase of the gas fraction or in the widening of the structure) or in the growth of the tip upward. The result of such complex dynamics is a complex path exhibiting more or less side branches depending on the value of $\chi$. Interestingly, on average, the gas occupies an elongated region whose radius of curvature near the outlet is $d \chi$, as shown by the numerical results.

From the dependence of the height $\Delta z$ and typical width $\sqrt{D_{\text {eff }} \Delta z}$, we can estimate the typical gaseous fraction $F$ inside the invaded region. Estimating the corresponding surface area $\sqrt{D_{\text {eff }}} h^{3 / 2}$, one gets from simple algebra $F \sim \chi^{1 / 4} / \sqrt{N}$, thus dependent on $\chi$ and on the injected volume (on $N$ ). The fraction $F$ slightly increases when $\chi$ increases (i.e., when the gravity effects are reduced and the side branching enhanced). In addition, denoting $w$ the typical relative variation of the pore size as proposed in Ref. [24], one can estimate further that $\sigma_{p} \sim$ $w \gamma / d$ and, thus, that $\chi \sim w \gamma /\left(\rho g_{\text {eff }} d^{2}\right)$. Thus, considering that the pore volume $v_{p} \propto d^{2}$ and taking into account the result obtained for $v_{z}$, we are taught that the maximum height reached by the gas within the granular does not depend on the grain size and scales like $V /\left(l_{c} \sqrt{w}\right)$ where $l_{c} \equiv \sqrt{\gamma / \rho g_{\text {eff }}}$. Thus, provided that the proposed estimate of $\chi$ is correct, for a given volume $V$, the maximum height is controlled by the capillary length $l_{c}$ and the relative width $w$, which account for the heterogeneity of the capillary overpressure.

In conclusion, we reported results of a numerical study which makes it possible to predict from the knowledge of one single control parameter $\chi$ the typical height, width, and gaseous fraction of the region invaded by a given volume of gas liberated at the base of an immersed granular bed. Such results could be of practical importance: For instance, gas can be trapped on purpose in an underground natural container below a granular slurry. Our results can help in predicting if the gas is likely to reach the free surface and escape the system if the container presents a defect (hole or fracture). The present study will be extended, from the theoretical point of view, to a slightly different geometrical situation, especially to the 3D case and, from the experimental point of view, to the case of a horizontal liquid flow.

\section{ACKNOWLEDGMENTS}

G.V. acknowledges a grant by CONICYT (Comisión Nacional de Investigación Científica y Tecnológica, Gobierno de Chile). The authors thank F. Vittoz for the technical support.
[1] T. Mörz, E. A. Karlik, S. Kreiter, and A. Kopf, Sediment. Geol. 196, 251 (2007).

[2] L. Naudts, J. Greinert, Y. Artemov, S. E. Beaubien, C. Borowski, and M. De Batist, Mar. Geol. 251, 253 (2008).

[3] I. N. Tsimpanogiannis and P. C. Lichtner, Phys. Rev. E 74, 056303 (2006).

[4] A. Mazzini, A. Nermoen, M. Krotkiewski, Y. Podladchikov, S. Planke, and H. Svensen, Mar. Petrol. Geol. 26, 1751 (2009).

[5] L. A. Porrit, R. A. F. Cas, and B. B. Crawford, J. Volcanol. Geotherm. Res. 174, 90 (2008).

[6] L. A. Porrit, R. A. F. Cas, and B. B. Crawford, J. Volcanol. Geotherm. Res. 178, 851 (2008).

[7] R. J. Brown, M. Field, T. Gernon, M. Gilbertson, and R. S. J. Sparksa, J. Volcanol. Geotherm. Res. 178, 847 (2008).

[8] R. Semer, J. A. Adams, and K. R. Reddy, Geotech. Geol. Eng. 16, 69 (1998).

[9] K. R. Newman et al., Earth Planet. Sci. Lett. 267, 341 (2008).

[10] H. Svensen, S. Planke, A. Malthe-Sørenssen, B. Jamtveit, R. Myklebust, T. R. Eldem, and S. Rey, Nature (London) 429, 542 (2004).
[11] M. Eden, in Proceedings of the Fourth Berkeley Symposium on Mathematical Statistics and Probability, edited by J. Neyman, (University of California Press, Berkeley, CA, 1961), p. 223.

[12] M. J. Vold, J. Colloid Sci. 18, 684 (1963).

[13] D. N. Sutherland, J. Colloid Interface Sci. 22, 300 (1966).

[14] T. A. Witten and Jr., L. M. Sander, Phys. Rev. Lett. 47, 1400 (1981).

[15] P. Meakin, J. Colloid Interface Sci. 96, 415 (1983).

[16] H. Martín, J. Vannimenus, and J. P. Nadal, Phys. Rev. A 30, 3205 (1984).

[17] M. Chaouche, N. Rakotomalala, D. Salin, B. Xu, and Y. C. Yortsos, Phys. Rev. E 49, 4133 (1994).

[18] A. Birovljev, L. Furuberg, J. Feder, T. Jøssang, K. J. Måløy, and A. Aharony, Phys. Rev. Lett. 67, 584 (1991).

[19] P. Meakin, J. Feder, V. Frette, and T. Jøssang, Phys. Rev. A 46, 3357 (1992).

[20] Z. Bo, D. Loggia, L. Xiaorong, G. Vasseur, and H. Ping, Eur. Phys. J. B 50, 631 (2006).

[21] R. Lenormand and C. Zarcone, Phys. Rev. Lett. 54, 2226 (1985). 
[22] F. Melo, F. Vivanco, C. Fuentes, and V. Apablaza, Int. J. Rock. Mech. Min. 44, 77 (2007).

[23] A. Nermoen, C. Raufaste, S. D. de Villiers, E. Jettestuen, P. Meakin, and D. K. Dysthe, Phys. Rev. E 81, 061305 (2010).

[24] G. Varas, V. Vidal, and J.-C. Géminard, Phys. Rev. E 83, 011302 (2011).
[25] L. Gostiaux, H. Gayvallet, and J.-C. Géminard, Granular Matter 4, 39 (2002).

[26] C. Chevalier, A. Lindner, M. Leroux, and E. Clément, J. NonNewtonian Fluid Mech. 158, 63 (2009).

[27] J. S. Selker, M. Niemet, N. G. Mcduffie, S. M. Gorelick, and J.-Y. Parlange, Transport Porous Med. 68, 107 (2007). 